\title{
Energy management for a commercial building microgrid with stationary and mobile battery storage
}

\author{
Yubo Wang $^{\mathrm{a}^{*}}$, Bin Wang ${ }^{\mathrm{a}}$, Chi-Cheng Chu ${ }^{\mathrm{a}}$, Hemanshu Pota ${ }^{\mathrm{b}}$ and Rajit Gadh ${ }^{\mathrm{a}}$
}

\author{
${ }^{a}$ Department of Mechanical Engineering, UCLA, Los Angeles, United States \\ b School of Engineering and Information Technology, University of New South Wales, Canberra, Australia \\ ${ }^{*}$ Corresponding author. Address: 44-120 Engr. IV, 420 Westwood Plaza, Los Angeles, CA 90095, USA. Email: \\ ybwang@ucla.edu
}

\begin{abstract}
This paper investigates the Demand Side Management (DSM) in a commercial building microgrid with solar generation, stationary Battery Energy Management System (BESS) and gridable (V2G) Electric Vehicle (EV) integration. Taking into consideration of a comprehensive pricing model, we first formulate a deterministic DSM as a mixed integer linear programming problem, assuming perfect knowledge of the uncertainties in the system. A two-stage stochastic DSM is further developed that addresses the stochastic nature in solar generation, loads, EV availabilities and EV energy demands. The proposed DSMs are validated with real solar generation, loads, BESS and EV data using sample average approximation. Detailed case studies show that the stochastic DSM outperforms its deterministic counterpart for cost saving for a wide range of prices, though at the expense of higher computational time. Computational results also demonstrate that moderate number of EVs helps to cut down the overall operation cost, which sheds light on the benefit of future large scale EV integration to smart buildings.
\end{abstract}

Keywords: Battery Energy Storage System (BESS); Demand Side Management (DSM); solar generation; Electric Vehicle (EV); Vehicle-to-Grid (V2G); smart buildings; stochastic programming.

\section{Nomenclature}

Sets, indices and naming rules

$\begin{array}{ll}\mathrm{H} \in \mathrm{Z} & \text { Horizon of a day } \\ \mathrm{s} \in \mathrm{Z} & \text { Scenario index } \\ \tau \in \mathrm{R} & \text { Duration of the timeslot } \\ \xi, V \in \mathrm{RH} & \text { Uncertainty set of solar generation and load } \\ \zeta \mathrm{i} \in \mathrm{RH} & \text { Uncertainty set of energy demand of EV } i \\ \delta i \in Z \mathrm{H} & \text { Uncertainty set of availability of EV } i \\ 1,0 \in \mathrm{ZH} & 1 \text { and } 0 \text { column vector } \\ \mathrm{Xs} \in \mathrm{ZH} / \mathrm{RH} & \text { Parameter/variable } \mathrm{X} \text { in scenario } s\end{array}$

Parameters 


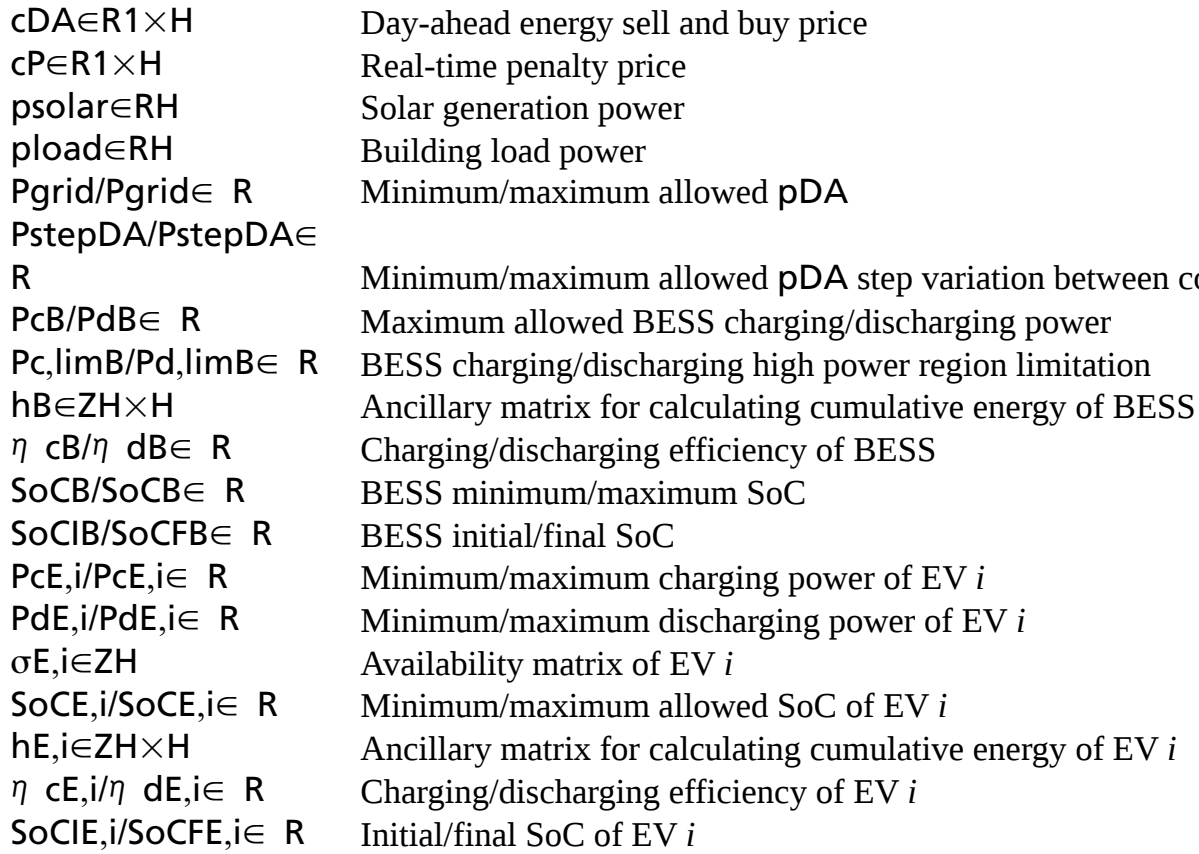

Decision variables

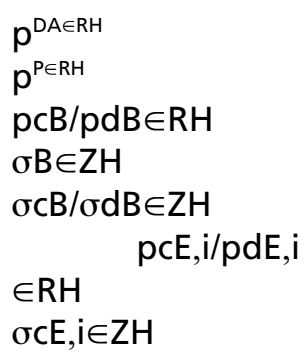

\section{Acronyms}

$\begin{array}{cl}\text { BESS } & \text { Battery Energy Storage System } \\ \text { DSM } & \text { Demand Side Management } \\ \text { EV } & \text { Electric Vehicle } \\ \text { KDE } & \text { Kernel Density Estimation } \\ \text { SAA } & \text { Sample Average Approximation } \\ \text { SoC } & \text { State-of-Charge } \\ \text { V2G } & \text { Vehicle-to-Grid }\end{array}$

\section{Introduction}

\subsection{Motivations}

Future smart buildings will incorporate an increasing renewable generation, dispatch units and storage devices, switching from traditional dumb consumptions to distributed and regulated counterparts. With continuous increase in Electric Vehicles (EVs) and solar penetrations for more than a decade [1, 2] unregulated EV charging together with intermittent solar generation are posing additional challenges on supply-demand balancing in smart buildings. 
Microgrids represent a vision for distributed generations and consumptions, enhancing the robustness of power grid and creating new ways of utilizing sustainable energy resources [3]. Facilitated by recent advances in Demand Side Management (DSM), renewable generation and loads are managed in response to variations in the price signal $[4,5]$. Coupled with the target of cutting down overall operational cost, DSM collaboratively addresses peak shaving and load shedding with physical and human comfort constrains [6-8].

The integration of EVs to households and commercial buildings is creating new challenges as well as opportunities [9, 10]. Combined with fluctuations in renewable generation, EVs' randomness in arrival and departure time, State-of-Charge (SoC) and energy demands, all add up to introduce larger uncertainties to the system. The emergence of EVs with Vehicle-to-Grid (V2G) capability has also restructured EVs' role from heavy loads to small-sized distributed virtual generators [11-13]. Hence, the DSM design in households and commercial buildings has been a subject of significant ongoing research.

\subsection{Literature review}

Previous research on DSM in households and commercial buildings has primarily focused on deterministic optimizations [14-17]. Two studies of DSM on a household with one EV and solar generation reported peak shifting with price incentives [14, 15]. Mixed integer linear programming optimization is used in both the papers to achieve minimum operation cost. Chabaud et al. [16] modeled a grid-connected residential building with renewables and battery storage, which confirmed the importance of energy storage device and diversity of renewable generation in cost saving. Furthermore, Shi et al. [17] studied the optimal energy management of residential units in a microgrid with a decentralized convex optimization method, which reduced computational time and preserved consumer privacy. These researchers assumed perfect knowledge of uncertainty and used deterministic optimization methods for DSM, without considering the loads, EVs and renewable generation's stochastic nature.

Much of the research in DSM has examined uncertainties caused by renewable generation and EVs with robust optimization [18-20]. Malysz et al. [18] considered uncertainties in loads and solar generation of a microgrid, and formulated the optimal control of a microgrid as a robust mixed integer linear programming problem. Bai et al. [19] evaluated a robust mixed integer quadratic programming optimization method for large scale V2G for EV aggregator taking into account EV instant power demand uncertainties. Zhang et al. [20] proposed distributed robust optimization algorithms for DSM with intermittent renewable generation in a microgrid. 
These methods addressed the uncertainties in the systems by estimating the worst case of the uncertainty sets, which might be conservative and resulted in high operational cost.

In [21-22], the wind generation uncertainties were modeled in a microgrid with probabilistic constrained stochastic programming. The operation cost minimizations were achieved with constraints of utilizing certain percentage of wind generation to meet minimum renewable utilization regulations. These papers considered the load and renewable uncertainties in the power grid, and addressed the stochastic behavior with uncertaintyaware stochastic optimization. However, these researchers did not consider how the randomness of EV's energy demands, arrival/departure time would interact with the intermittent renewable generation, which if managed improperly, will increase the burden of a microgrid.

Apart from the mixed integer linear programming, mixed integer quadratic programming and convex optimization used in deterministic optimization, robust optimization and stochastic optimization mentioned above, heuristic-based DSM has captured researcher's attention [23-25]. Adaptive neuro-fuzzy inference system [23], fuzzy logic [24], and $\theta$-krill herd [25] are some of the examples of heuristic based DSM. Although heuristic based DSM allows operators to achieve multiple optimization goals at the same time, compared to traditional mixed integer linear programming, mixed integer quadratic programming and convex optimization, the heuristic methods cannot be solved with standard solvers and hence may result in longer computational time.

Together with many paper not cited, existing papers have made sound contributions to DSM in households, commercial buildings. However, existing research either failed to consider the effect of EVs' integration in DSM or did not capture uncertainties in renewable generation, loads and EV modeling. Furthermore, though DSM in households and commercial buildings shares some of the similarities, a commercial building typically has more EVs introducing larger uncertainties on demand side. Hence, DSM of a grid-connected commercial building with renewable generation and EVs needs to be reexamined in details.

\subsection{Scope, contributions and organization}

This paper jointly studies DSM in a commercial building microgrid with solar generation, building loads, Battery Energy Storage System (BESS) and EVs. We consider a comprehensive pricing model and targets at maintaining a low operation cost while utilizing solar generation, stationary BESS and mobile EV storage as much as possible. We first deduct a deterministic DSM and formulate it into a mixed integer linear programming problem. Taking into consideration the stochastic behaviors of solar generation, building loads and EVs, we 
further develop DSM with two-stage stochastic programming. Sample Average Approximation (SAA) Monte Carlo simulation is used to get numerical results of the proposed DSMs.

The major contributions of this paper are threefold. Firstly, this paper describes the first research in which stochastic nature of individual EV is modeled and studied together with other uncertainty elements in a commercial building microgrid setting. Secondly, to validate the proposed DSMs, this paper uses real solar generation, building load, EV availabilities and EV energy demands data to evaluate the performance of the proposed methods. Finally, this paper shares detailed comparisons between deterministic DSM and its stochastic counterpart, in terms of price variation, BESS sizing and EV numbers.

The remainder of the paper is organized as follows: Section 2 describes and models the studied system. It is followed by presenting and analyzing numerical results in Section 3. Section 4 draws conclusions and discusses future researches.

\section{System descriptions and modeling methodologies}

In this section, we first introduce the system we are studying and show the challenges of DSM under uncertainties. Then, we develop a deterministic model with the estimations on uncertainties. It is followed by modeling the uncertainties in the deterministic model and formulating the DSM into a stochastic model.

\subsection{System descriptions}

This paper studies a grid-connected commercial building microgrid that a BESS, several EVs and large volume solar generation are integrated to the building. The system architecture of the grid-connected commercial building microgrid is shown in Fig.1. As the primary target of this paper is to analyze how stationary and mobile storage can help in DSM in the context of uncertainties on both supply and demand sides, we intentionally separate EVs and BESS from building load. Other forms of stationary storage exist in commercial buildings such as ice storage unit and heat storage unit [26]. However, BESS allows bidirectional power flow, which adds more flexibility to the DSM design. We evaluate the BESS's impact on DSM, but same modeling technique can be applied for analysis of other stationary storages. 


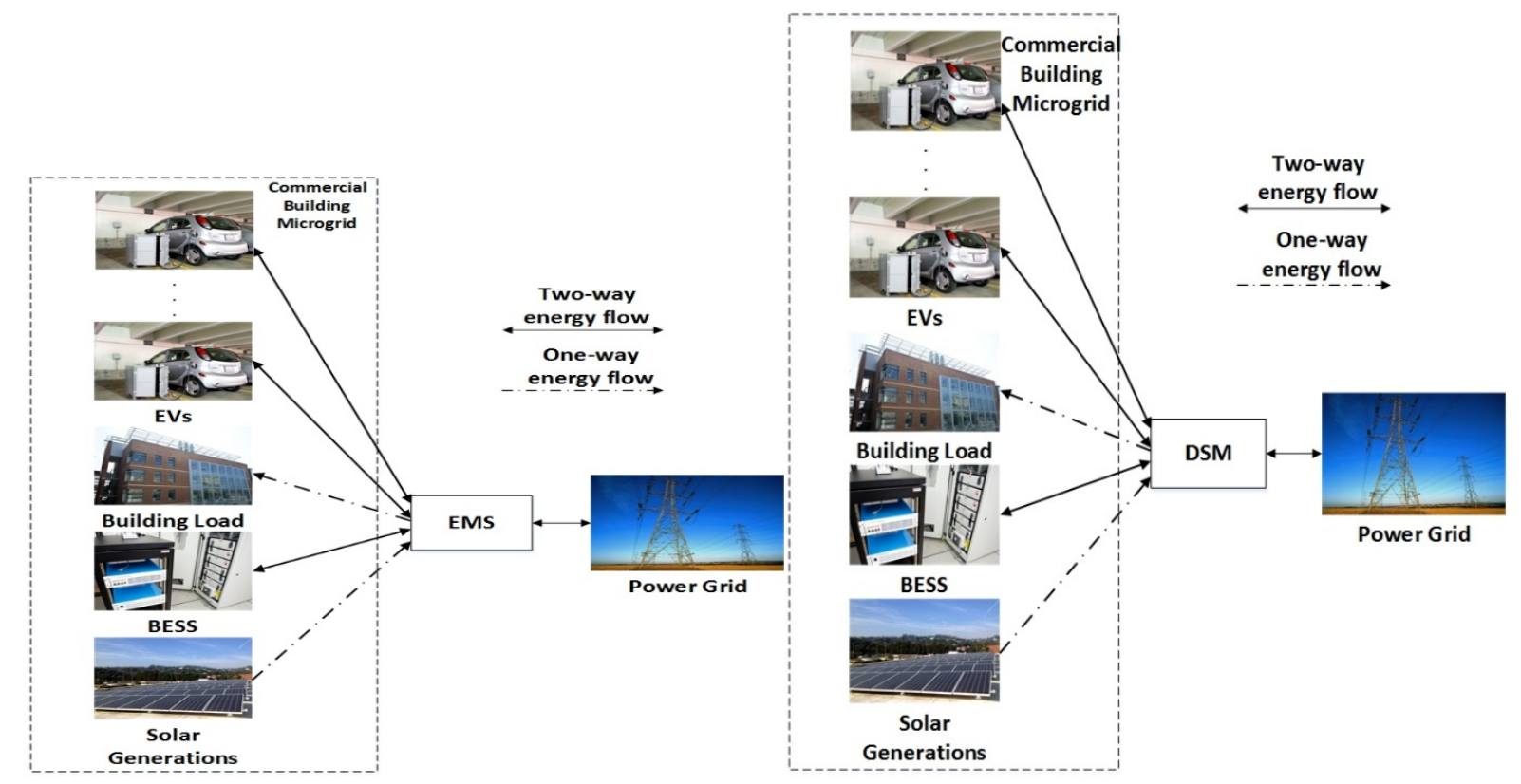

Fig.1 Architecture of a grid-connected commercial building microgrid

We take building load data from [27] and $35 \mathrm{~kW}$ capacity solar generation data from [28]. The typical weekdays and weekends building loads are shown in Fig.2(a) and sunny day solar generation is shown in Fig.2(b). Looking at the data we notice that, for the building load, weekday and weekend patterns are distinct. Even though building load in weekdays/weekends follow similar patterns, it is affected by random user behavior, outside temperature, etc., resulting in some fluctuation in load data. Similarly, in Fig.2(b), it is shown that in a typical sunny days, solar generations will also have small fluctuations, resulting from cloud shedding and temperature change. The typical weekdays building load and sunny day solar generation are shown in Fig.2. 

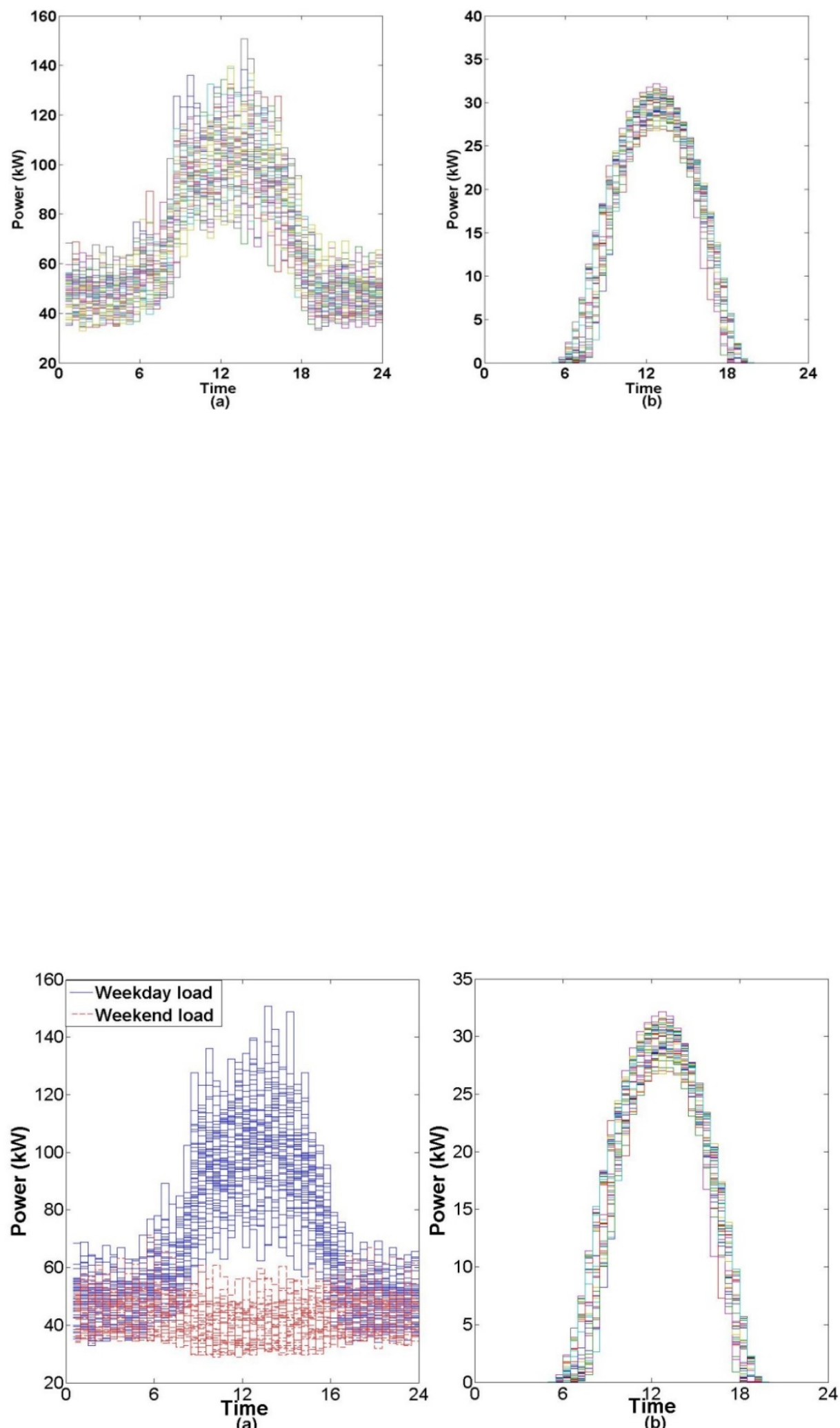

Fig.2 Power variations (a) building load on weekdays and weekends (b) solar generation in sunny days 

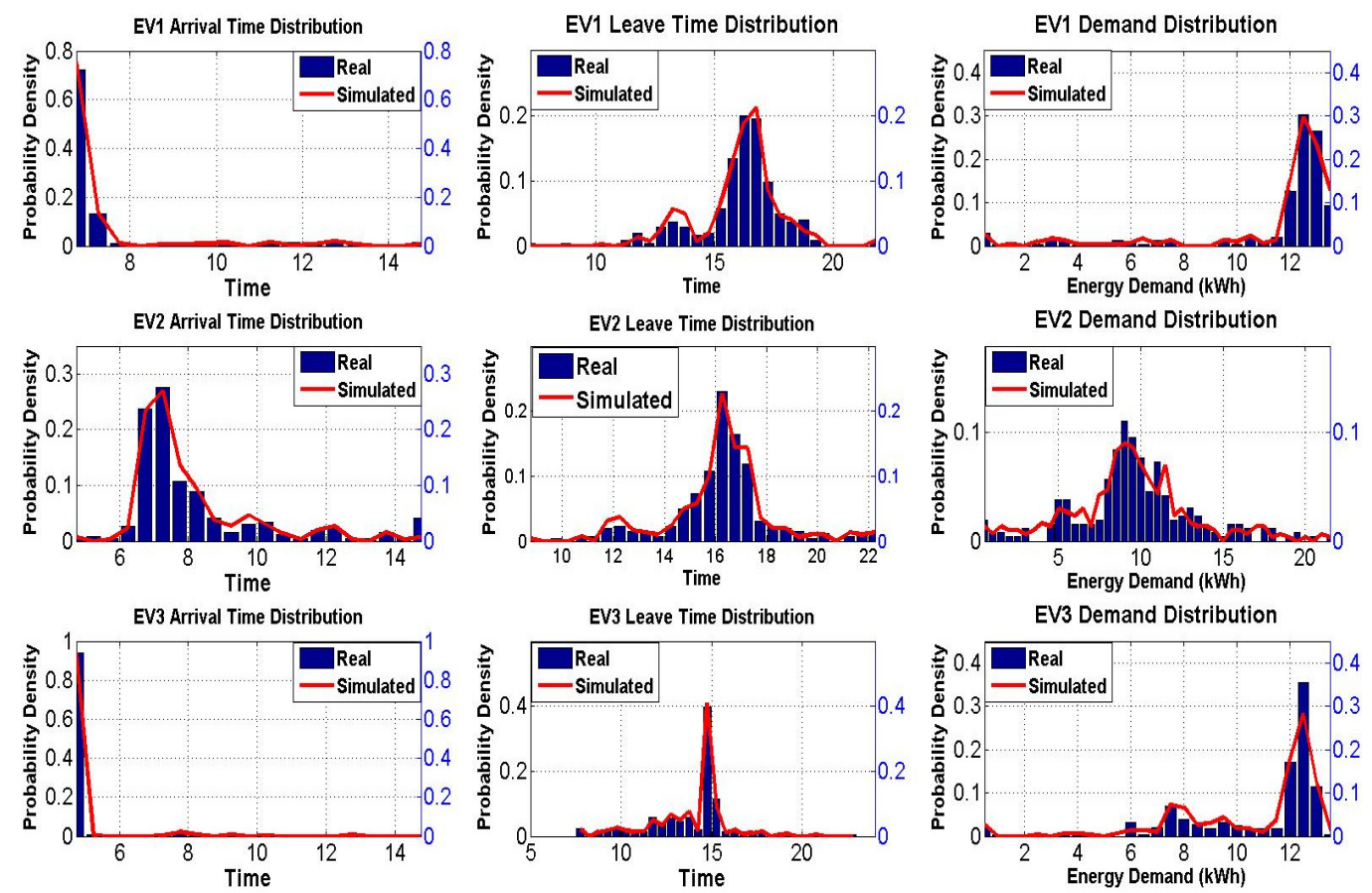

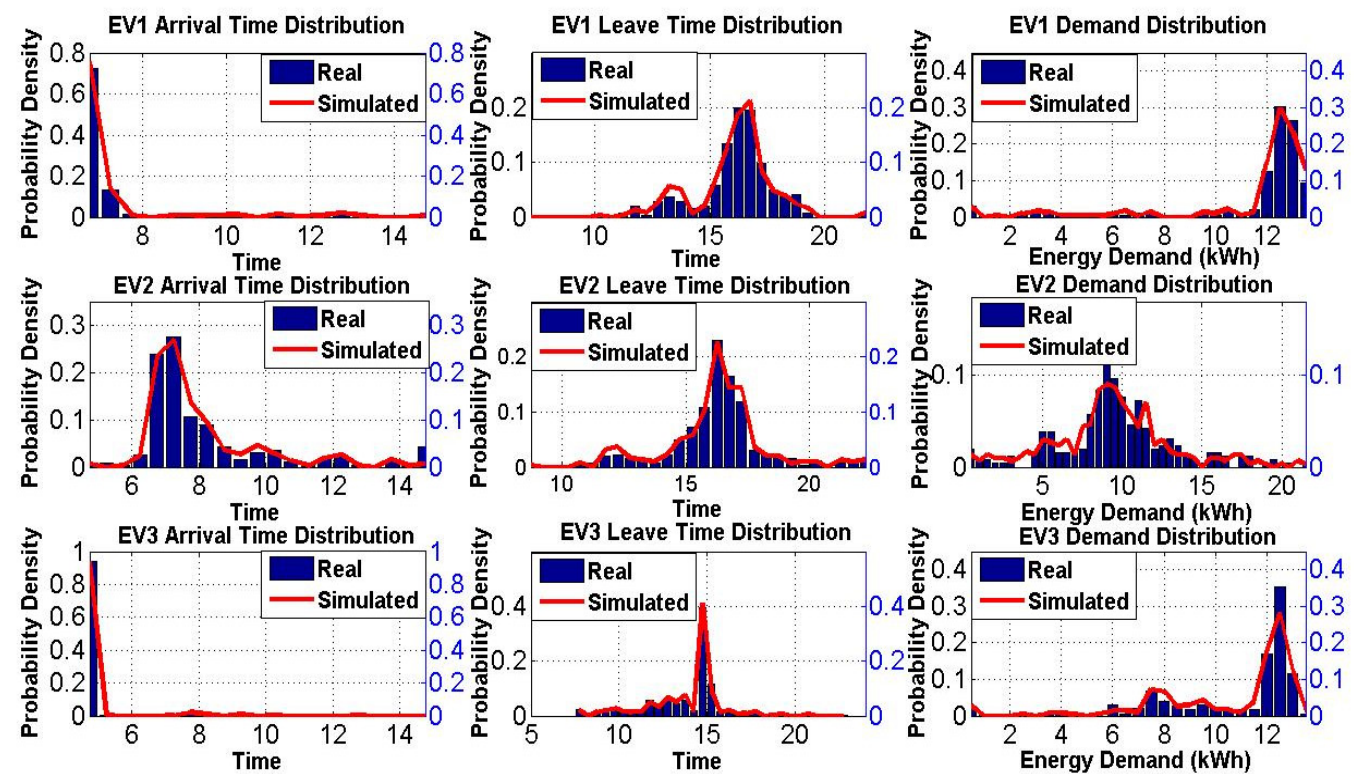

Fig.3 Three EV users’ distinct behavior

Three anonymous real EV users from UCLA charging networks [29-30] are studied. They drive a Mistubishi MiEV, a Nissan Leaf and a Nissan Leaf respectively, all with CHAdeMO port for V2G [31]. They come on campus on a daily basis during weekdays and park their car in the parking structure attached to the campus building. The distributions of arrival time, departure time and energy demands shown in Fig.3 are extracted from over a year's charging records. And Fig.3 is further discussed in Section 3.1 on scenario generations. Note that although the data is collected from the UCLA charging networks, these users typically finish their charging long before departure, thus the data is available for the V2G study in the proposed method.

\subsection{Deterministic DSM}

In this subsection, we present the detailed modeling of each component shown in Fig. 1 with deterministic models. The inputs to the model are price signals, solar generations, building loads, BESS sizing, EV energy demands and availabilities. We make detailed analysis of each input in Section 3.3.

\subsubsection{Pricing model and cost function}

In this paper, we consider a comprehensive pricing model with day-ahead transactions cost and real-time penalty cost. The commercial building microgrid, needs to provide day-ahead energy purchase/sell decisions before the start of the next day. Furthermore, as the day-ahead buy and sell transactions are based on the estimations of the energy consumption for the next day. A mismatch always exists between the day-ahead predicted energy 
demands and the real-time consumption, which is compensated with a penalty. The compensated power can be either positive or negative as long as it satisfies the power balance which is discussed later.

The model we use in this paper is the slotted model, assuming variables stay constant between decision intervals т. We assume that the price is perfectly known, and the size of the generation and consumption of the commercial building microgrid does not change the price. The economical compensations to EV users for V2G is a very complicated problem including proper business model [32] and battery degrading cost [33], which are not in the scope of this paper and will be covered in future research. The optimal DSM cost function is formulated as follows:

$$
\min C^{\mathrm{DApDA} \tau+\mathrm{CPIPP}(\xi, v, \delta, \zeta) \mid \tau}
$$

The operation cost includes day-ahead buying and selling cost and real-time penalty cost. Given that uncertainties exist in renewable generation, loads, EV availabilities and energy demands, we use estimation of each uncertainty in the deterministic model. The estimation method is discussed in Section 3.3.

\subsubsection{Power balancing and grid constraints}

At any time instance of the slotted model, the power flowing into the commercial building microgrid should be always equal to the power flowing out, which is modeled as follows:

$$
i=1 \mathrm{MpdEV}, i \delta i, \zeta i+p s o l a r \xi+p d B+p D A=i=1 \mathrm{MpcEV}, i \delta i, \zeta i+p l o a d v+p c B+p^{p \xi, v, \delta, \zeta}
$$

where $M$ is the number of EV. Note that uncertainties of load and solar generation are represented by psolar $\overline{\text { }}$ and pload $v$. The uncertainties in EV energy demands and availabilities are represented by $\delta i, \zeta$ i. pDA, pdB, pcB are all decision variables that are needed to be determined day-ahead, while solar generation, building loads and EV availabilities and energy demands will not be exactly known until the next day. In this model, we address the uncertainties in a deterministic perspective, i.e., using estimations for each uncertainty in DSM.

We need further constraints to control the behavior of power injected to the power grid.

$$
\text { Pgrid } \leq p D A+p^{P} \leq \text { Pgrid }
$$

$$
\text { PstepDA } \leq(p D A) j+(p D A) j+1 \leq \text { PstepDA }, \quad j=1,2, \ldots, H-1
$$


where (pDA)j denotes row $j$ of pDA. Eq.3 limits the maximum power that the commercial building microgrid can buy from and sell to the grid due to line capacity. Eq.4 regulates the variation of power in day-ahead market, so that the shaping of day-ahead power is smoother and more predictable to the utilities.

\subsubsection{BESS modeling}

The modeling of BESS includes power, energy and state constraints as follows:

$$
\begin{aligned}
& 0 \leq \mathrm{pcB} \leq \mathrm{PcB} \sigma \mathrm{B} \\
& 0 \leq \operatorname{pdB} \leq \operatorname{PdB}(1-\sigma B) \\
& \mathrm{Pc}, \lim \mathrm{B} \sigma c \mathrm{~B} \leq \mathrm{pcB} \leq \mathrm{Pc}, \operatorname{limB} 1-\sigma c \mathrm{~B}+\mathrm{PcB} \sigma c \mathrm{~B} \\
& P d, \lim B \sigma d B \leq p d B \leq P d, \operatorname{limB} 1-\sigma d B+P d B \sigma d B \\
& (\sigma \subset B) j+(\sigma c B) j+1 \leq 1, \quad j=1,2, \ldots, \mathrm{H}-1 \\
& (\sigma d B) j+(\sigma d B) j+1 \leq 1, \quad j=1,2, \ldots, H-1 \\
& \text { SoCFB }=S \circ C l B+(h B) \text { end }(p c B \eta c B-p d B \eta d B)
\end{aligned}
$$

$\mathrm{hB}$ is an ancillary matrix that is used for calculating the SoC at each decision interval $\tau$; $(\mathrm{hB})$ end denotes the last row of the $\mathrm{hB}$ matrix. Eq.5 and Eq.6 limit the maximum charging and discharging power for BESS so that it does not exceed physical limitations. It is reported in [18] that BESS can operate in high power charging/discharging region for peak demand, however for only a limited time. Eq.7 -Eq.10 ensure that the BESS will not consecutively operate in high power charging/discharging region consecutively for more than one time slot. Integer decision variables $\sigma \mathrm{B}, \sigma \mathrm{CB}, \sigma \mathrm{dB}$ are introduced in Eq.5-Eq.10 for regulating the BESS operation status. Taking into consideration that deep discharge will affect the Lithium-ion battery's lifecycle [34], Eq.11 constrains the SoC of BESS so that it does not violate physical constraints and operate in deep discharge region. In the end, Eq.12 defines the final SoC for BESS after a day’s operation.

\subsubsection{EV modeling}

The modeling of EV has to consider the uncertainties in demand, arrival time, and departure time. We develop the model with uncertainties bore in mind using estimation for each uncertainty variable as follows: 


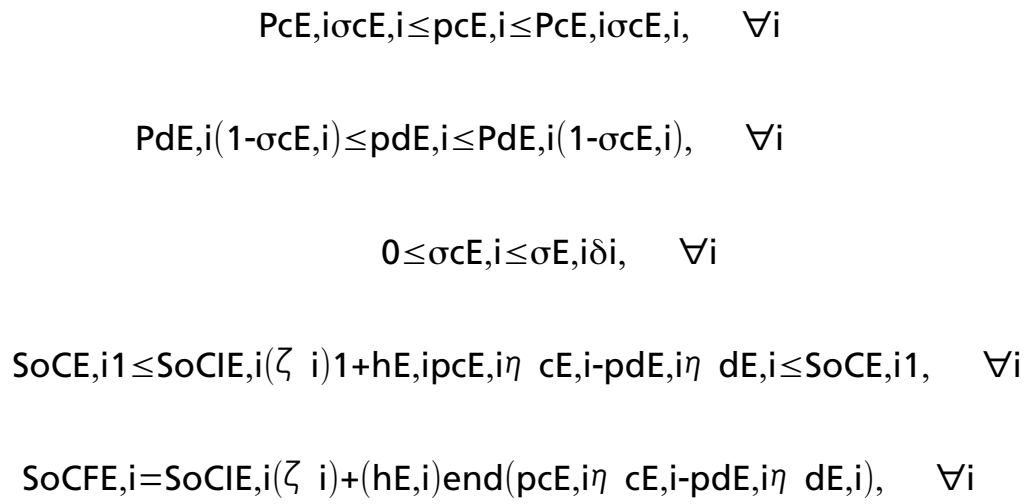

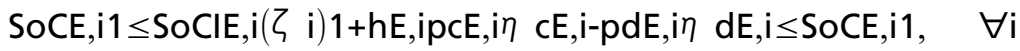$$
\text { SoCFE,i=SoCIE,i( } \zeta \text { i })+(h E, i) \text { end }(p c E, i \eta \quad c E, i-p d E, i \eta \quad d E, i), \quad \forall i
$$

Similar to $\mathrm{hB}, \mathrm{hE}, \mathrm{i}$ is ancillary matrix that for calculating the SoC of EV $i$ at each decision interval $\tau$ ( $h E, i)$ end denotes the last row of the hEV,i matrix. Eq.13 and Eq.14 model the limits of charging and discharging power for each EV. Without loss of generality, integer decision variables are introduced in Eq.13 and Eq.14 are for modeling the charging and discharging losses in Eq.16 and Eq.17. Also, integer variables are introduced due to the fact that both charging and discharging need to reach minimum power, making the power output of EVs discontinuous [10]. The uncertainty of EV availabilities is reflected in $\sigma \mathrm{E}$,idi in Eq.15, which actually models the stochastic arrival and departure time of each EV. The uncertainty of energy demand for each EV is captured with SoCIE,i( $(\zeta)$ in both Eq.16 and Eq.17. As these uncertainties exist in each EV, the corresponding decision variables pcE,i and pdE,iare naturally subject to uncertainties, which are linked to Eq.2. It is noted that in deterministic model we recognize the existence of EV behavioral uncertainties. We use estimations for each uncertainty, which is discussed in Section 3.3.

\subsection{Stochastic DSM}

The deterministic model is a favorable solution to DSM in commercial building microgrid however not without problems. Looking at Fig.2 and Fig.3, building load and solar generation follow some trends, however still present fluctuations due to unpredictable factors such as random user behavior and cloud shedding. On the other hand, EV arrival and departure time and energy demands are subject to the EV owner behaviors, which are hard to predict. In addition, as the EV number increases, the charging/discharging of EVs will become a major load/generation to commercial buildings in the future. How to make decisions on day-ahead energy transactions and BESS operation under the context of larger uncertainties becomes challenging, which gives rise to stochastic DSM. 


\subsubsection{Problem formulation}

Instead of optimizing cost function under uncertainties with estimation in Eq.1, we now optimize the expectation of the cost function. The problem is then formulated into a two-stage stochastic programming problem as follows:

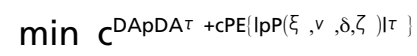

$\mathrm{p}^{\mathrm{DA}}$ are first-stage decision variables while $\mathrm{p}^{\mathrm{P}}$ are second-stage decision variables. We are optimizing first-stage day-ahead transactions in the context of second-stage expectation. For more details, see Appendix 1.

Given the stochastic nature of renewable generation, loads, and EV availabilities and demands, we may want to extract the distribution of each uncertainty and formulate the expectation in Eq.18 analytically. However, even if we may be able to extract the distribution of each random variable, the requirement to know joint distribution of a number of uncertainties in the optimization problem such as Eq.1-Eq.17 make the problem hard to solve. Instead we seek to use SAA and solve the optimization problem numerically.

SAA is a Monte Carlo simulation technique that serves as a statistical inference of the original problem (in this case Eq.18). The idea behind it is to generate a large pool of samples of uncertainties based on their empirical distributions where each sample is called a scenario. Then solve the second-stage problems with each scenario before averaging them out to approximate the original problem. For a detailed discussion, see [35].

Without loss of generality, it is assumed that we generate $N$ independent scenarios. Each scenario is one possible realization of the uncertainties, which includes solar generation, building loads, EV availabilities and energy demands. Hence, the power balance and EV constraints, which involve uncertainties, are updated as follows:

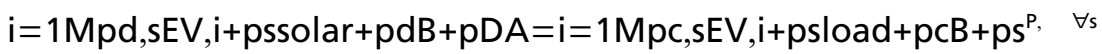

$$
\begin{aligned}
& \text { PcE, ioc,sE, } i \leq p c, s E, i \leq P c E, i \sigma c, s E, i, \quad \forall i, \forall s \\
& \operatorname{PdE}, \mathrm{i}(1-\sigma c, s \mathrm{~s}, \mathrm{i}) \leq \mathrm{pd}, \mathrm{sE}, \mathrm{i} \leq \mathrm{PdE}, \mathrm{i}(1-\sigma c, \mathrm{sE}, \mathrm{i}), \quad \forall \mathrm{i}, \quad \forall \mathrm{s} \\
& 0 \leq \sigma c, s \mathrm{E}, \mathrm{i} \leq \sigma s \mathrm{E}, \mathrm{i}, \quad \forall \mathrm{i}, \forall \mathrm{s} \\
& \text { SoCF, sE,i=SoCl,sE,i+hsE,iend(pc,sE, i } \eta \quad c E, i-p d, s E, i \eta \quad d E, i), \quad \forall i, \quad \forall s
\end{aligned}
$$


Eq.19 shows the power balance within each scenario. The subscript $s$ denotes scenario number s among $N$ generated scenarios. Eq.22 presents the realization the EV availabilities uncertainty by generating osE,i in each scenario. Similarly, Eq.23 and Eq.24 address EV energy demands uncertainty by SoCl,sE,i and SoCF,sE,i. It is noted that Eq.19-Eq.24 should hold within each generated scenario and for all $N$ scenarios. Consequently, the stochastic DSM is formulated as follows:

$$
\begin{aligned}
& \min c^{\mathrm{DApDA} \tau+1 \mathrm{Ns}=1 \mathrm{NcPlpsP} \mid \tau} \\
& \text { s.t. (3)-(12), (19)-(24) }
\end{aligned}
$$

Using Eq.25, we approximate the original problem (Eq.18) with SAA. It has been reported in [36] that a twostage stochastic programming with integer first-stage and second-stage variables can be approximated by SAA. Furthermore, SAA exponentially approximates the true problem as scenario size $N$ increases.

On feasibility side, the problem is always feasible as long as the EV constraints can be met. The worst case is when an EV user submits an energy demand that needs charging from arrival to departure. However, it is rarely seen for the EV owners we studied. On grid side, there is no upper and lower bounds for ps ${ }^{p}$ so that the power balance constraints can always be met in Eq.19.

\subsubsection{Sample Average Approximation bounds estimation}

SAA is a statistical inference of the original problem. We generate scenarios before solving the SAA problem. As the scenario is generated based on empirical distribution, naturally, we will have slightly different SAA results if we solve the SAA problem for multiple times. This gives rise to evaluation of the SAA, in which we need to examine how closely the SAA optimum approximates that of the original problem. We follow the methods in [36] to find out the upper and lower bounds for the SAA problem. The algorithm is tabulated in Algorithm 1. Before start of iteration, we set $k=1$. Then we generate $N$ i.i.d. scenarios based on the scenario generation method described in Section 3.1. We solve the stochastic DSM (25) using the generated $N$ samples.

Then, we store the first-stage variables $\mathrm{p}^{\mathrm{DA}, \mathrm{pcB}, \mathrm{pdB}, \sigma \mathrm{\sigma B}, \sigma \mathrm{\sigma B}, \sigma \mathrm{dB}}$ as $\mathrm{x}$ and optimum as opt(k). It is followed by generating $N^{\prime}$ i.i.d. scenarios, typically, $\mathrm{N}^{\prime} \gg \mathrm{N}$. We solve (25) with the fixed stored first-stage variables $\mathrm{x}$ minimizing over only second-stage decision variables which includes $p s^{p, p c, s E, i}$ and $p d, s E, i$. The optimum for the scenario $N^{\prime}$ is denoted as optk. We iterate the procedure for $K$ times where $K$ is a predefined number. The optimization gap can be estimated using opt and opt. 


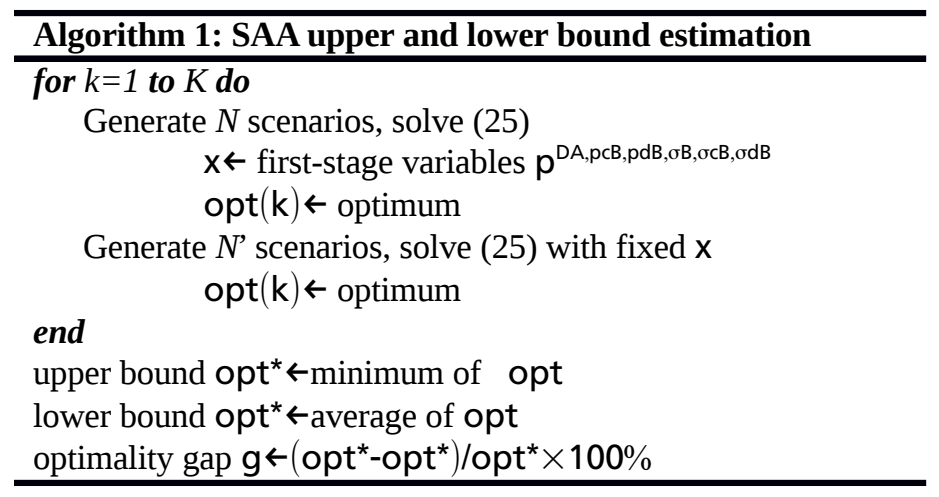

\section{Computational results and analysis}

In this section, we validate the proposed DSMs. We first describe how we generate the scenarios based on the collected data. It is followed by a case study of the system and analysis on computational results.

\subsection{Scenarios generation}

We first classify the historical load data into weekdays and weekend, and solar generation data into sunny, cloudy and rainy groups. Solar generation and load behave differently between groups while showing similarity within each group. Before running DSM, we pick corresponding solar generation and load data from historical data based on the forecast/knowledge of the next day. In this paper, we investigate the performance of the DSM on sunny weekdays. However, the methodology used in this paper can be also applied to weekdays/weekends with other weather conditions. We capture the distribution of the real data shown in Fig.2 and Fig.3 with Kernel Density Estimations (KDE) [37]. We use the KDE modeled distribution to generate scenarios and compare them with real distribution of EV user behavior shown in Fig.3. The comparison demonstrates a close match between the KDE simulated data and the real distribution.

Table 1 summarizes some of the key parameters used in the following computational case study. We use $\tau=0.5$ h. The BESS data is taken from a real BESS in the University of California, Los Angles, laboratory. EV maximum charging power is a typical number for charging in Level II circuits [38]. For EV discharging power, we refer to the industrial product in [39]. In the following case study, EV energy demands are recognized as the energy required to fully charge the corresponding EV from its initial SoC. Moreover, we set initial SoC of BESS equal to its final SoC after a day’s operation. Fig.4 shows the day-ahead price and real-time penalty price from CAISO. We increase the penalty price by twenty five percent to see the performance of the two DSMs. The penalty price sensitivity is studied in discussion section, showing that the DSMs proposed in this paper work 
generally well in a wide range of prices. The proposed DSMs are solved with commercial solver Gurobi [40] on a PC with 3.1GHz CPU and 16 GB RAM.

Table 1. Key parameters

\begin{tabular}{llll}
\hline Item & Value & Item & Value \\
\hline BESS battery size & $70 \mathrm{kWh}$ & Mitsubishi MiEV battery size & $16 \mathrm{kWh}$ \\
BESS initial and final SoC & 0.6 & Nissan Leaf battery size & $24 \mathrm{kWh}$ \\
BESS maximum charging power & $40 \mathrm{~kW}$ & EV maximum charging power & $6.6 \mathrm{~kW}$ \\
BESS maximum charging power & $20 \mathrm{~kW}$ & EV maximum discharging power & $2.0 \mathrm{~kW}$ \\
Min/max allowed pDA & $150 \mathrm{~kW}$ & Min/max allowed pDA variation & $15 \mathrm{~kW}$ \\
\hline
\end{tabular}



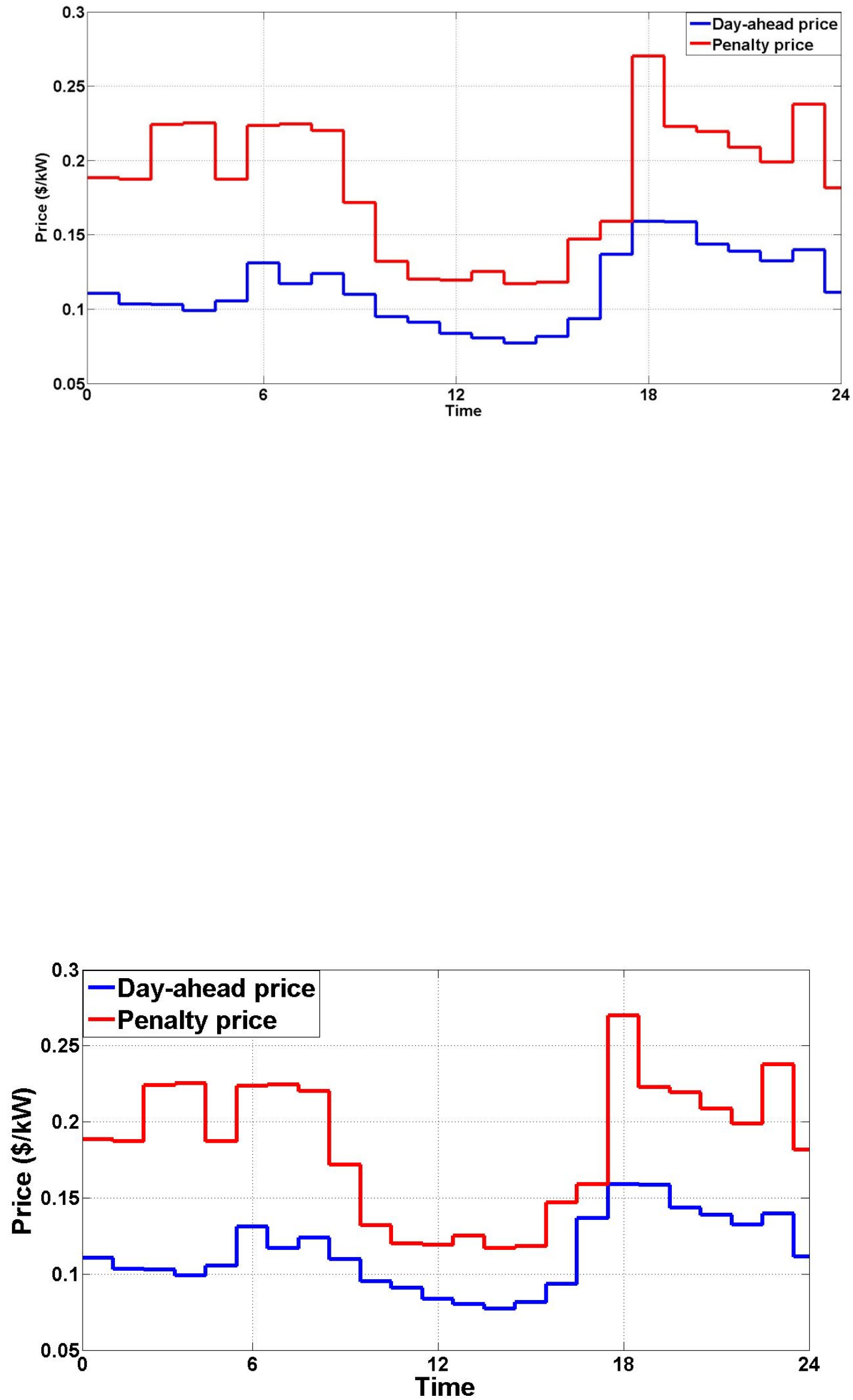
Fig.4 Price for a 24 hour period from CAISO

\subsection{Case study}

We apply the proposed stochastic DSM to a microgrid that is made up of a grid-connected commercial building with weekday building load. Fig.5 presents computation results of the building profile of a day. From the figure we can see that pDA variation is limited by the maximum step PstepDA and PstepDA. Consequently dayahead power is smoother and easier to follow on utility side. Looking at BESS power output, we see the high power region restrictions work correctly so that the BESS will not keep operating in excessive high power regions. Solar generation, $\mathrm{p}^{\mathrm{p}}$ and loads are plotted for one typically scenario among many of the generated scenarios in Fig.5. We observe that the real-time power mismatch between the day-ahead planning and real-time operation, which is compensated by $p^{p}$, is relatively small compared to day-ahead bidding power $p^{\text {DA }}$. It allows utilities to better plan generations and reduces real-time uncertainties.

Fig.6 shows the EV profile of the same scenario. We can see that three EVs arrive and depart at different times. They arrive with different SoCs and leave with fully charged batteries. Depending on the energy demand of each EV, they operate in V2G mode to support the building grid. Fig.5 together with Fig.6 demonstrates the correctness of the proposed stochastic DSM. 


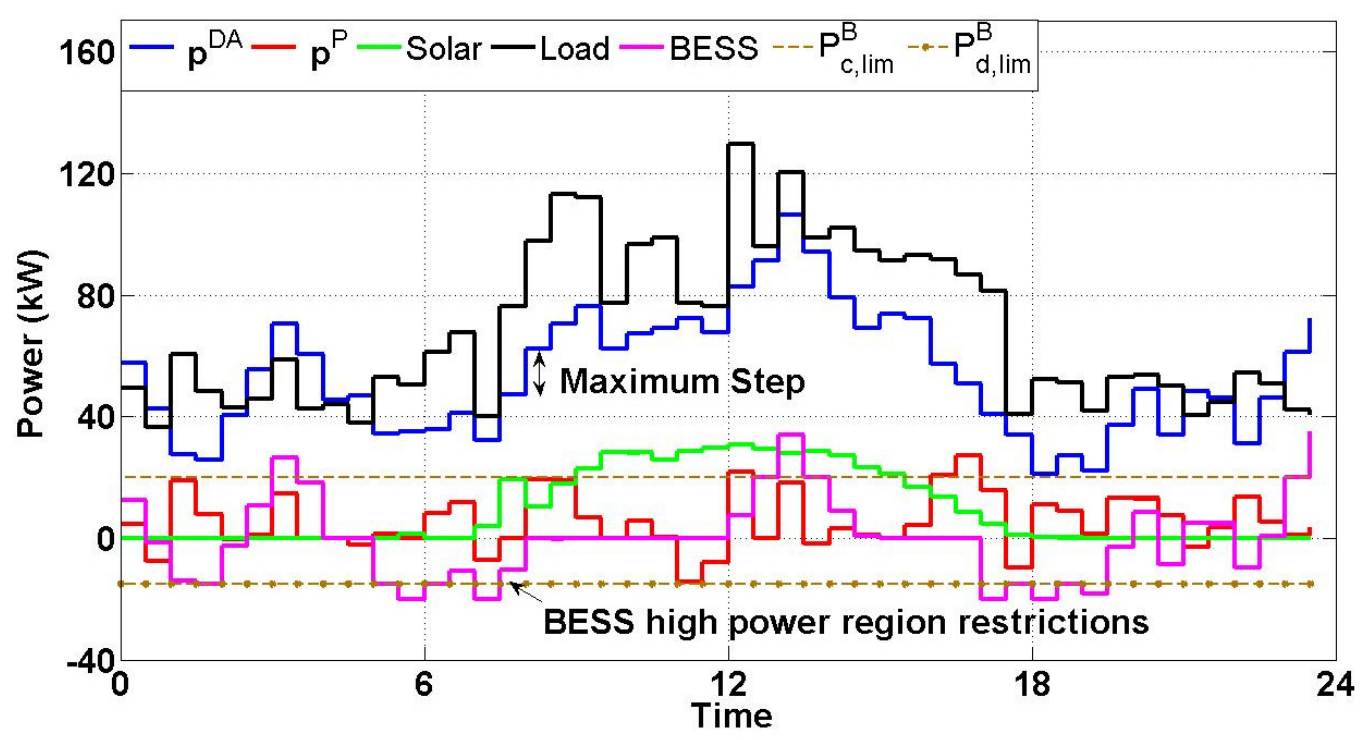




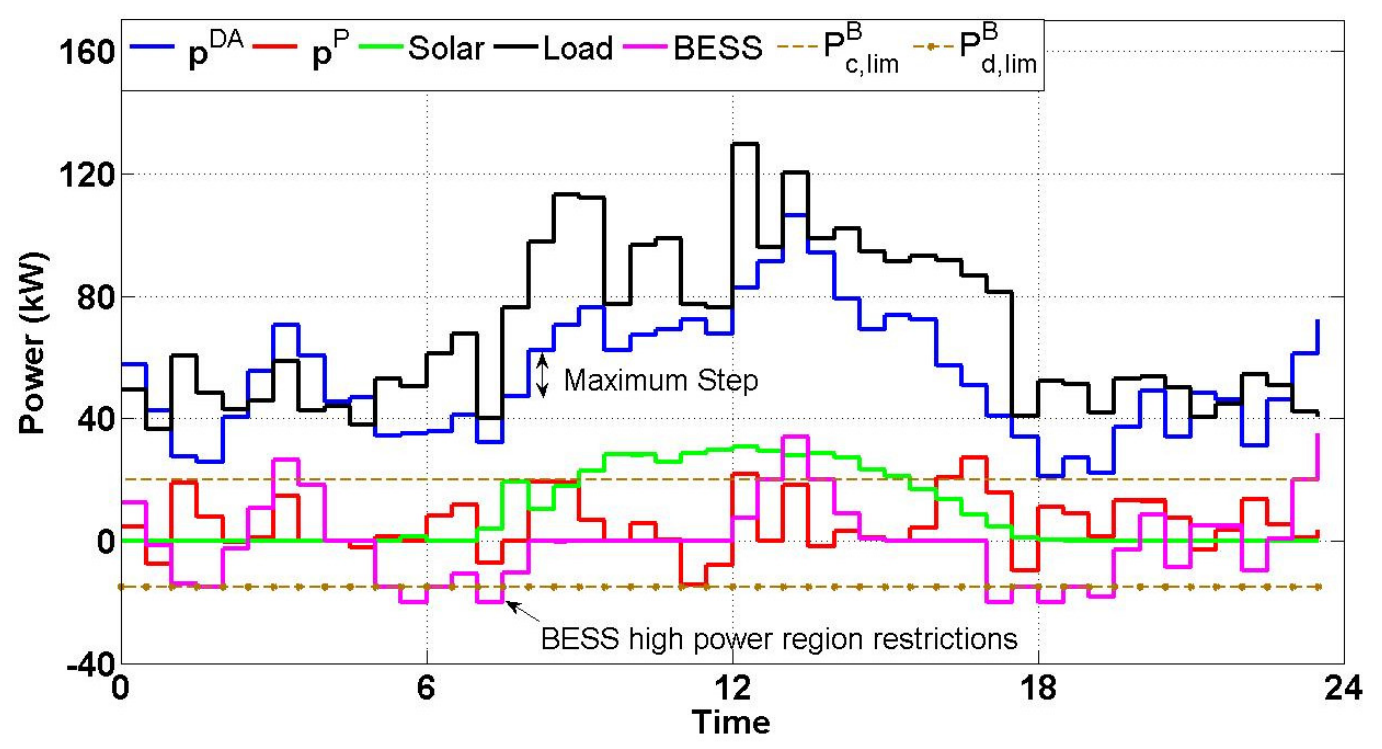

Fig. 5 Building profile of a day 


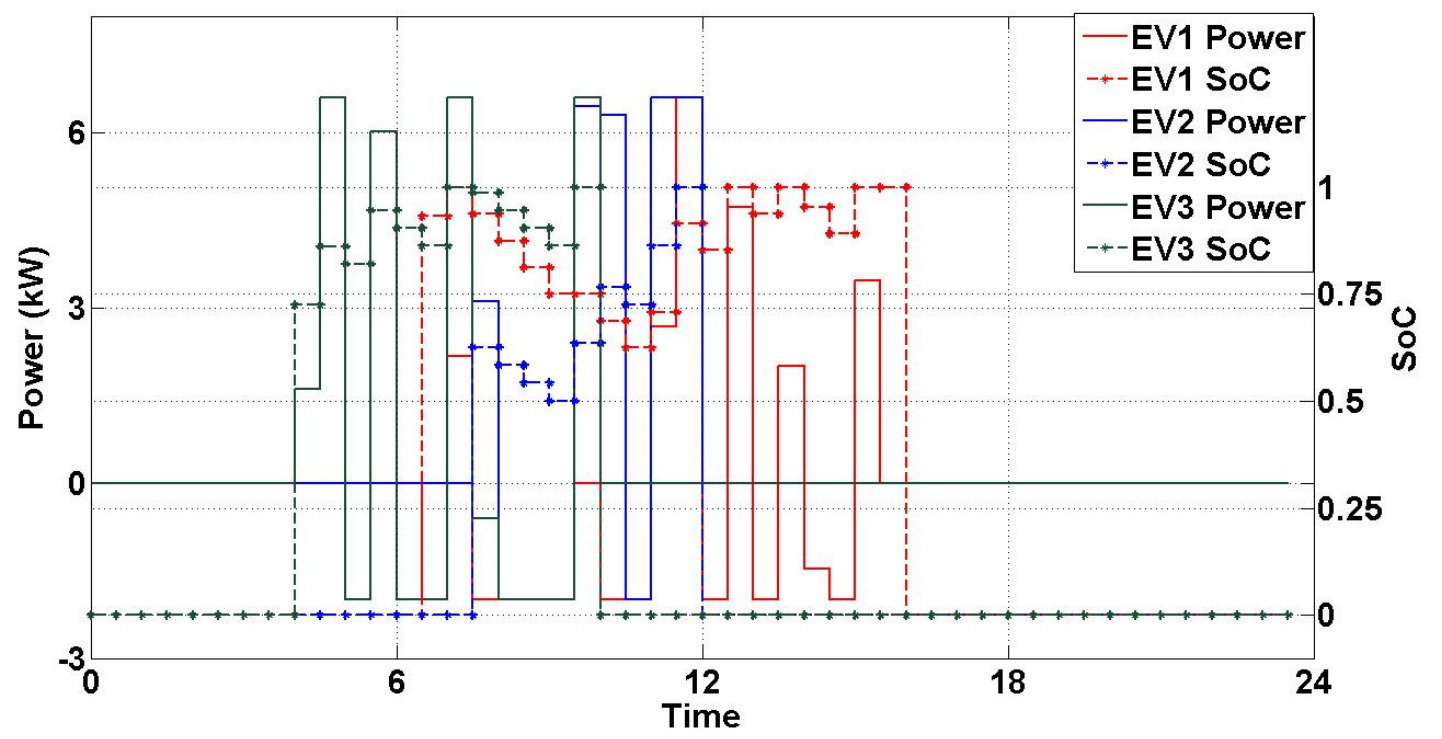




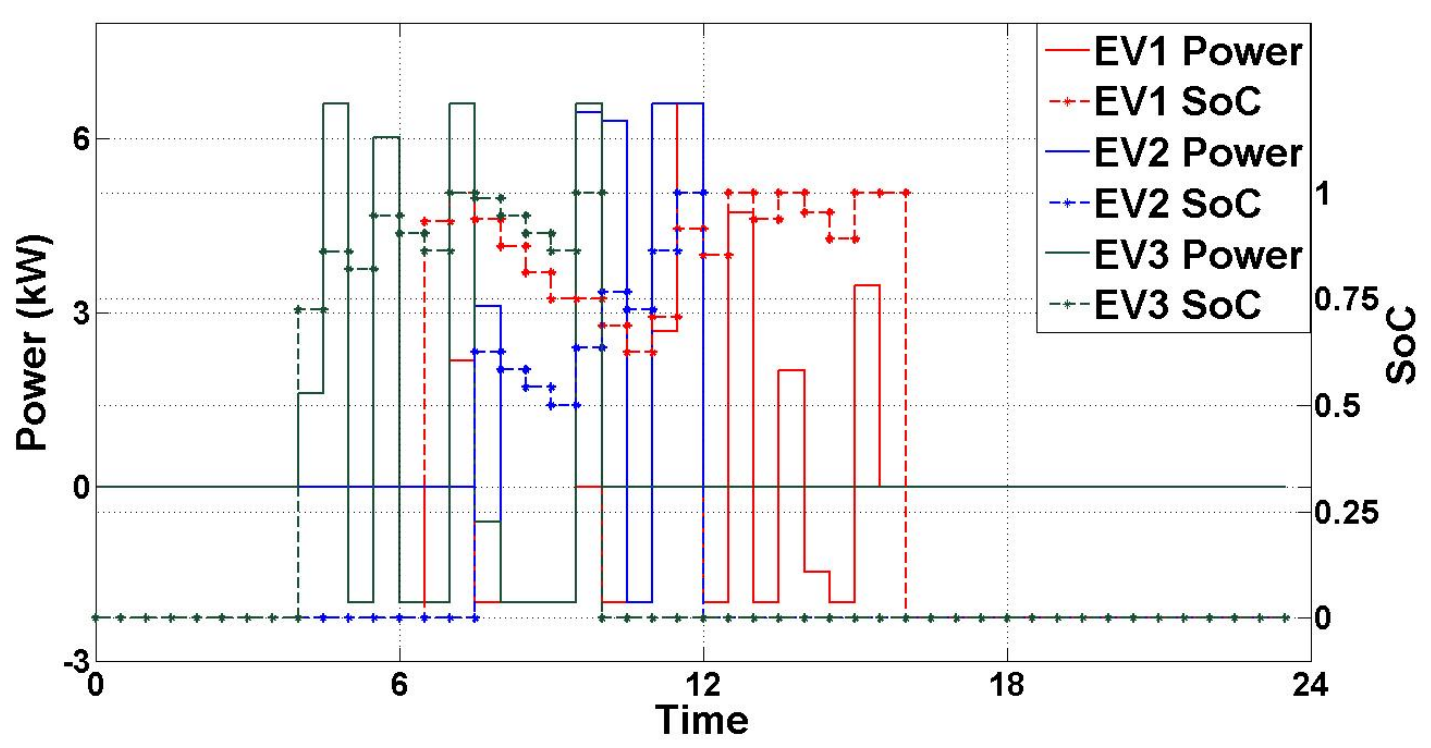

Fig.6 EV profile of a day

Besides examining the building and EV profile, we want to find out how closely SAA approaches the original problem. We follow the steps in Algorithm 1 and choose $K=10$ to test the optimality gap, which is defined in Algorithm 1. From the results summarized in Table 2, we can find out that in general the problem is tightly bounded. The computational time includes time consumed for both sample size $N$ and $N^{\prime}$, in which time for $N$ ' is much larger than $N$. Looking at the results we may safely conclude that using the lower bound of $N / N^{\prime}=50 / 500$, which is calculated by solving the 50 scenario problem for 10 times, is good enough for producing accurate results. We use the lower bounds estimated with $N=50$ in the following simulations of stochastic DSM. It consumes much less time compared with the estimation using the upper bound.

Table 2. Optimality gaps estimations of SAA

\begin{tabular}{ccccc}
\hline $\mathbf{N}^{\prime} \mathbf{N}^{\prime}$ & $\begin{array}{c}\text { Upper } \\
\text { bound }\end{array}$ & $\begin{array}{c}\text { Lower } \\
\text { bound }\end{array}$ & $\begin{array}{c}\text { Optimality } \\
\text { Gap }\end{array}$ & $\begin{array}{c}\text { Computational Time } \\
\text { (s) }\end{array}$ \\
\hline $\begin{array}{c}\mathbf{2 0 / 2 0} \\
\mathbf{0}\end{array}$ & 171.53 & 170.51 & $0.60 \%$ & 1088.7 \\
$\begin{array}{c}30 / 30 \\
\mathbf{0}\end{array}$ & 171.52 & 170.38 & $0.67 \%$ & 2297.1 \\
$\begin{array}{c}\mathbf{5 0 / 5 0} \\
\mathbf{0}\end{array}$ & 171.74 & 171.02 & $0.427 \%$ & 5607.8 \\
\hline
\end{tabular}

\subsection{Discussions}

1) Deterministic vs. stochastic. It is interesting to examine the difference between the proposed deterministic DSM and stochastic DSM. In the proposed deterministic DSM, we use average value of each uncertainty variables as their estimations. We verify the performance of both deterministic and stochastic DSMs with 50 
generated scenarios for 10 times. We compare how different load can affect the performance of the two studied DSMs by using the weekdays and weekends building load previously plotted in Fig.2. The simulation settings are kept the same as it is in Section 3.2 except for building load. The comparison results are tabulated in Table 3. It demonstrates that the proposed stochastic DSM outperforms its deterministic counterpart no matter under weekdays or weekend building load. In weekday cases, stochastic DSM shows (179.44-171.09)/171.09=4.88\% outperformance, while (113.34-107.25)/107.25=5.67\% outperformance in weekends. The outperformance in operation cost saving is at the expense of higher computational time. For a DSM that handles day-ahead planning, a longer time is tolerable.

In order to determine the solar capacity's influence on the proposed DSMs, we study the performance of two DSMs under wide solar power generation variation. We run numerical experiment with exactly same setting as it is in Section 3.2, except for varying the solar capacity. The results are presented in Fig.7. The same solar generation data is used for the solar power generation, however, it is multiplied by a scaling factor ranging from 0.75 to 4 for each studied case. With the current setting, a scaling factor of 4 makes the peak solar generation slightly larger than the peak building load. Simulation results in Fig.7 demonstrate that the stochastic DSM outperforms deterministic DSM in a wide range of solar generation capacity. Relative performance in Fig.7 is defined as the operational cost reduction of the stochastic DSM compared to its deterministic counterpart, divided by the operational cost of stochastic DSM. The relative performance curve shows a constantly increasing spread over the scaling factor. This is an expected result stemming from the larger uncertainty growing solar generation introduces to the system, which the deterministic DSM fails to capture. On the computational cost side, the time for the two DSMs is similar to the weekday results tabulated in Table 3. In terms of cost analysis, [41] reported the latest averaged solar installation costs of $\$ 4.40 / \mathrm{W}$ in California. Using this data, the $35 \mathrm{~kW}$ solar installation studied in this paper requires an investment of more than $\$ 1.5$ million. Without strong government subsidy, solar integration to the smart building is still very expensive.

Table 3. Comparisons of deterministic DSM and stochastic DSM

\begin{tabular}{lcccc}
\hline & $\begin{array}{l}\text { Deterministic } \\
\text { DSM cost (\$) }\end{array}$ & $\begin{array}{l}\text { Stochastic } \\
\text { DSM cost (\$) }\end{array}$ & $\begin{array}{l}\text { Deterministic D M M } \\
\text { computational time (s) }\end{array}$ & $\begin{array}{l}\text { S t o ch a t i c D S M } \\
\text { computational time (s) }\end{array}$ \\
\hline Weekdays & 179.44 & 171.09 & 4.23 & 248.83 \\
Weekends & 113.34 & 107.25 & 5.14 & 316.88 \\
\hline
\end{tabular}



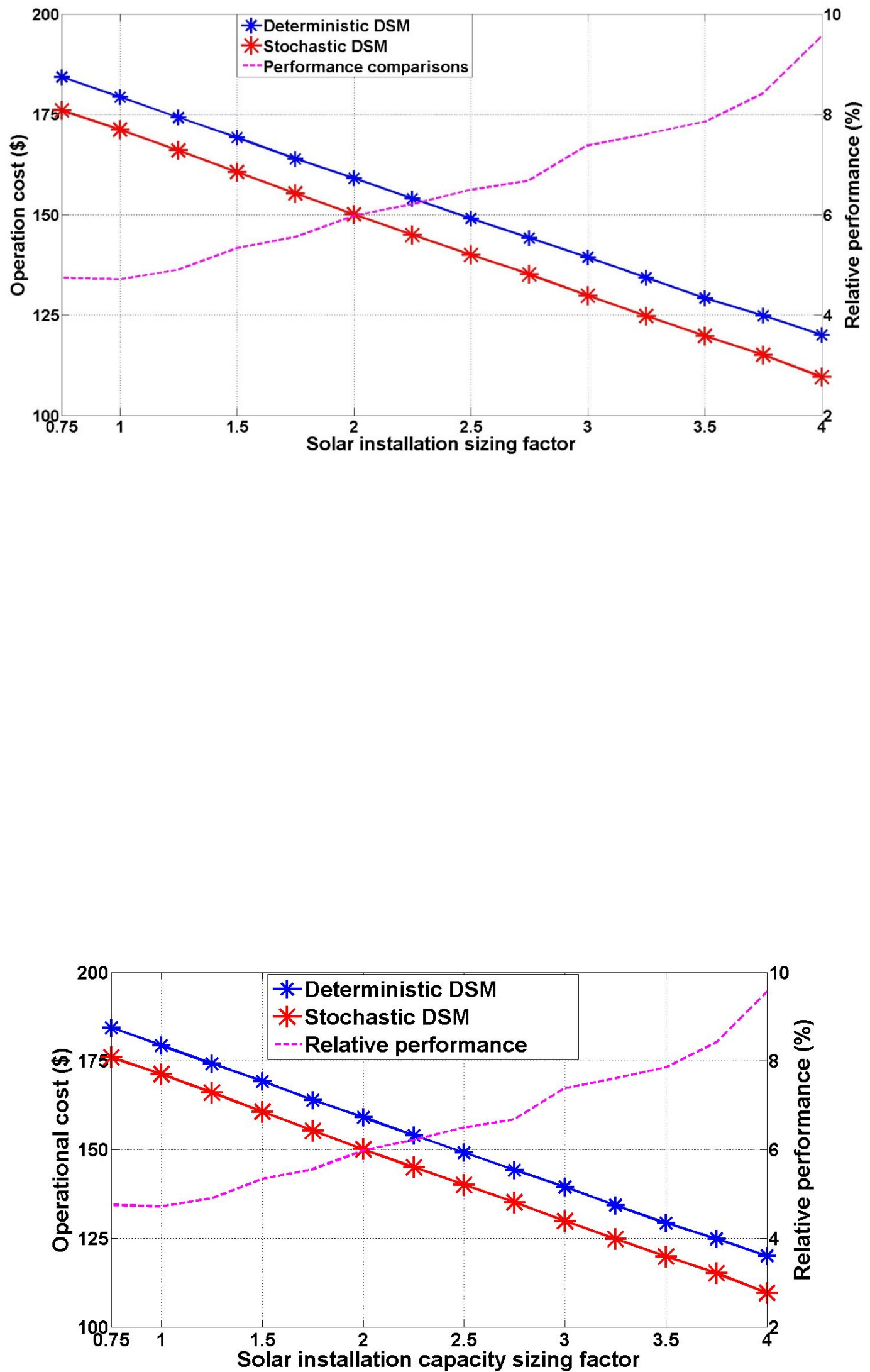

Fig.7 Comparisons of deterministic DSM and stochastic DSM with changing solar installation sizing 
2) Price sensitivity. We further analyze the DSMs sensitivities when price is changed. We fixed the day-ahead price and add variations to the real-time penalty price. Table 4 shows the comparisons of operational cost change of both deterministic and stochastic DSM given $\pm 25 \% \quad c^{P}$ variations. There are several interesting observations: a) Compared to the $25 \%$ change in penalty price, the operational cost in either deterministic or stochastic DSM stays relatively stable. This is expected as the majority of the power supplied to the building microgrid is from $c^{\mathrm{DA}}$. b) Stochastic DSM generally outperforms its deterministic counterpart, especially when the penalty price is lower than current level. The better performance with lower penalty price results from the fact that the DSM tends to buy more power from real-time penalty market when $c^{p}$ is lower. And as DSMs buy more power from the real-time penalty market, stochastic DSM has a better estimation on power should be purchased from realtime penalty market compared to its deterministic counterpart. In general, it can be projected that in reality when $c^{p}$ varies in a small range (less than $10 \%$ ), the stochastic DSM will continue to outperform its deterministic counterpart with steady performance.

Table 4. Comparisons of operational cost under $c^{p}$ variation

\begin{tabular}{lccc}
\hline & $\mathbf{7 5 \%} \mathbf{C}^{\mathbf{P}}$ & $\mathbf{1 0 0 \%} \mathbf{C}^{\mathbf{P}}$ & $\mathbf{1 2 5 \%} \mathbf{C}^{\mathbf{P}}$ \\
\hline Deterministic & 170.98 & 179.44 & 185.99 \\
DSM & & & \\
Stochastic DSM & 161.17 & 171.09 & 178.65 \\
Improvements & 9.81 & 8.35 & 7.33 \\
\hline
\end{tabular}

3) Impact of stationary storage. Stationary BESS is an investment that integrates to the commercial building microgrid for cost savings. We investigate how the change in capacity and power of BESS affect the overall performance of a stochastic DSM and the preliminary results are shown in Fig. 8. The blue asterisks show the impacts of BESS capacity changes alone where the capacity is multiplied with a sizing coefficient. The blue line is a linear regression curve fitting of the blue asterisks which shows a close match. Similarly, red asterisks show the impacts when power and capacity of BESS are sized together, and red line is its corresponding curve fitting. The slight mismatch at sizing coefficient equals to one between the blue and red asterisk is a natural result from SAA computational error. Experimental results show that cost is cut down with BESS capacity increase, and the cost is further reduced with the help of sizing up the BESS power. However, an increase in BESS power means investing larger power inverters which will add up to the cost. Study in [42] showed that though Li-ion battery price is decreasing rapidly, it still costs $\$ 0.55 / \mathrm{Wh}$. And study in [43] reported $\$ 0.65 / \mathrm{W}$ averaged cost in power 
electronics. Looking at the results presenting in Fig.8, a 100\% increase in BESS capacity and power results in a 171.27-167.57= $\$ 3.70$ cost saving per day. At the current capacity and power of BESS, such an increase indicates \$64,500 additional investment in hardware only, not to mention the neglected installation and software costs. Without subsidy and other incentives, such an investment seems not fruitful. 

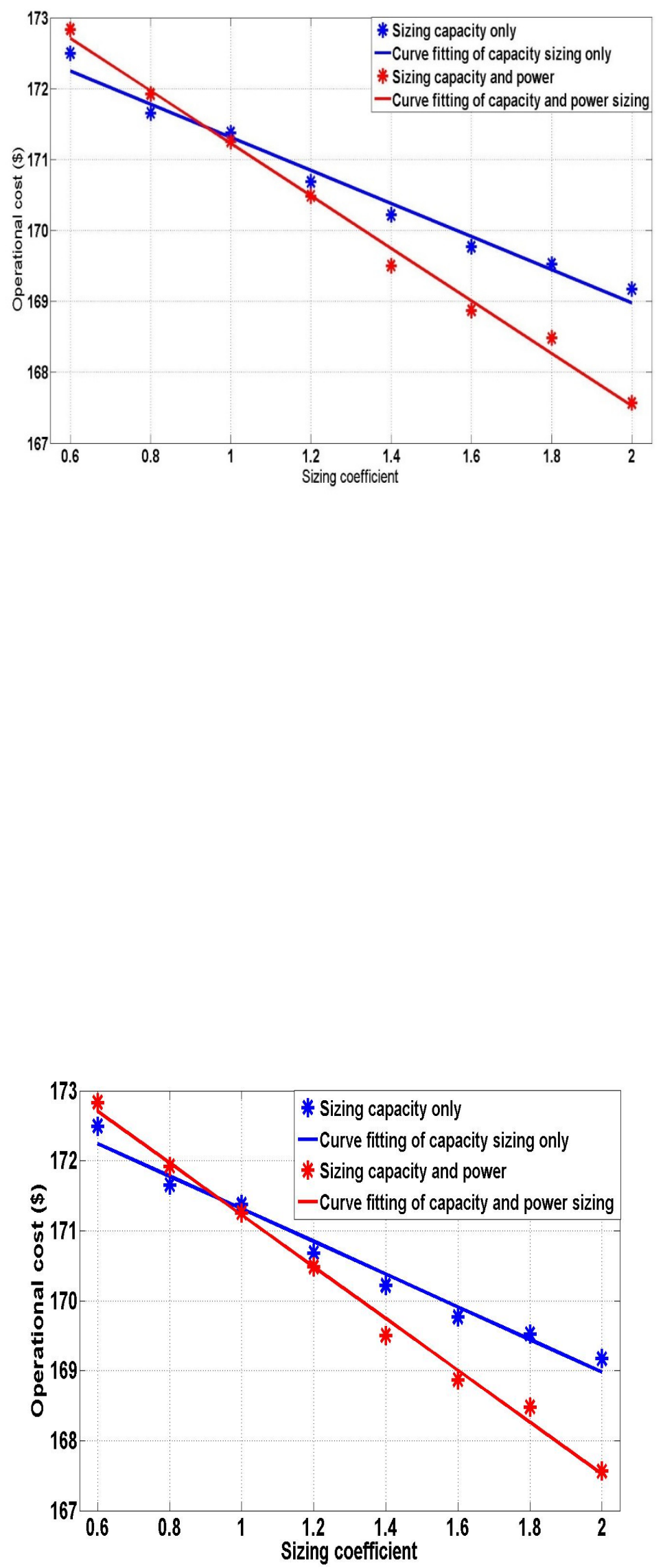
Fig.8 Sizing of BESS and its impacts

4) Impact of mobile storage. We further study the impact of change in the number of EVs on the operational cost. We duplicate the three EV profiles and create identically independent scenarios from $0 \mathrm{EV}$ to $15 \mathrm{EVs}$. The computational results of the stochastic DSM are presented in Fig. 9. We simulate a case with the original settings and a case with discharging power increased by $200 \%$. Surprisingly, the overall operational cost first goes down with increasing number of EVs after which the operation cost increases again. It can be explained as follow: with small number of EVs, they first help the commercial building microgrid to shave peak demands. However, as EV number continues to increase, the peak shaving cost reduction cannot compensate for the additional EV energy demands and therefore the cost rebounds. On the other hand, the cost reduction of increasing the discharging power from $2 \mathrm{~kW}$ to $6 \mathrm{~kW}$ requires an increase in power of the bidirectional off-board EV chargers. According to the $\$ 0.65 / \mathrm{W}$ power electronics cost reported in [43], the incremental cost of power electronics to upgrade for a $2 \mathrm{~kW}$ to $6 \mathrm{~kW}$ capacity is roughly $\$ 2,600$, a number much smaller compared to the cost of increasing the capacity and power rating of BESS. Compared to BESS, the batteries in EVs do not require additional investment from the building operator, the investment in increasing EV charger power capacity seems a smarter decision. The fast increase in computational time as a result of increased EV number stems from the additional EV related decision variables and constraints. Consequently, instead of the individual EV models currently used, we may need to develop lumped EV models, such as V2G-Sim [44], to reduce the computational time when EV number is large. 

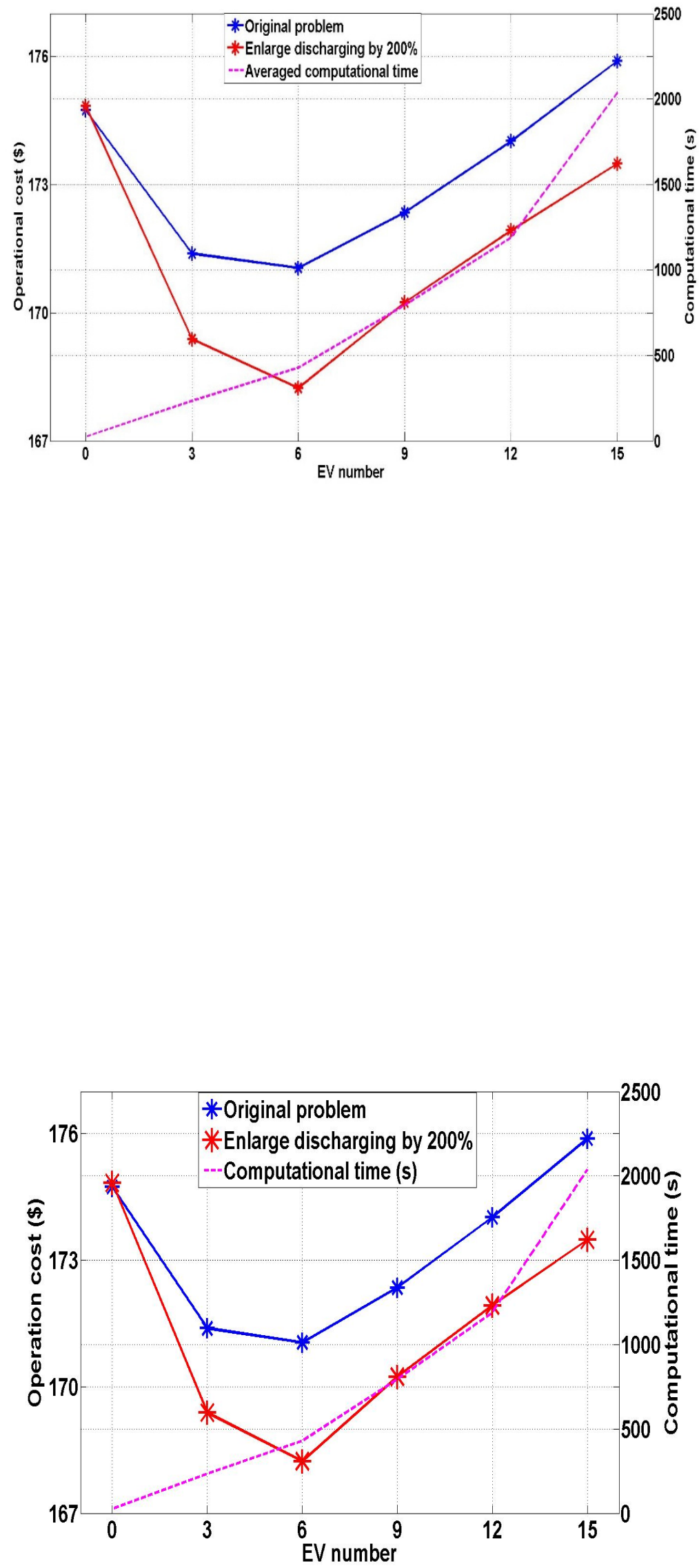
Fig.9 EV number variation and its impacts

\section{Conclusions and future research}

Several interesting observations are made based on the case studies presented in this paper. Firstly, the proposed stochastic DSM outperformances its deterministic counterpart for different load patterns, wide ranges of solar installation capacity and electricity prices. Although the computational time for the stochastic DSM is higher, it is still practical for the offline applications studied in this paper. Secondly, even solar installation saves electricity bill and decrease the building's dependence on traditional centralized generations, the cost is still very expensive. Thirdly, an increase in the capacity and power of BESS generally helps to cut down the operational cost of the commercial building microgrid. However, compared to the cost reduction, the additional hardware investments do not show a good return if there are no rebate incentives. Finally, an increase in the number of EVs in a commercial building microgrid surprisingly helps to cut down the operation cost at first after which the cost starts to increase. The additional cost reduction imposed by larger discharge power helps the building manager to decide the power rating of off-board bidirectional chargers.

The numerical analysis in this paper conforms that the moderate number of gridable EV integration is beneficial to cost reduction of commercial building microgrids. Stationary BESS as an optional energy storage system is economically expensive and part of its cost reduction functionality can be achieved by properly managed mobile storage (EVs). Furthermore, the deterministic DSM developed in this paper is a powerful tool for building managers to further cutting down operational cost when the commercial building microgrid is integrated with solar generation and EVs. In general, the methodology used in stochastic DSM can be applied to other commercial building microgrids that integrate a variety of other storage systems and renewable generation for better management under the context of uncertainty.

A number of problems need to be studied in the future. Firstly, the lumped commercial building load used in this paper can be decomposed into individual loads. It is projected to have a better performance at the expense of higher computational cost. Secondly, some uncertainties in the system may need to be re-examined. One example of such reevaluations is the variation in EV numbers when system continuous to scale up. Finally, when EV number continues to increase, a lumped EV model needs to be developed to avoid excessive computational time increase resulting from additionally introduced decision variables and constraints. 


\section{Acknowledgement}

This work has been sponsored in part by a grant from the LADWP/DOE fund 20699 \& 20686, (Smart Grid Regional Demonstration Project)

\section{Appendix}

Brief introduction to two-stage stochastic linear programming

A typical two-stage stochastic linear programming can be formulated as minimization over expectation [35]:

$\operatorname{mincT} x+E Q x, \delta$ s.t. $A x \leq B C x=D$

where $x$ are the first-stage decision variables, $\delta$ denotes the uncertainty sets, EQx, $\delta$ is the optimum of the second-stage problem:

$\min$ qTys.t. $T x+W y \leq h$

where $y$ are the second-stage variables, and uncertainty sets $\delta=\{\mathbf{q}, \mathbf{T}, \mathbf{W}, \mathbf{y}\}$. The two-stage problem can be interpreted as optimizing first-stage plus the expectation of the second-stage problem when uncertainties are not known. Second-stage problem on the other hand describes the optimization problem when the uncertainties are revealed.

\section{Reference}

[1] International Energy Agency, “Global EV Outlook,” 2013, [Online] Available: http://www.iea.org/publications/globalevoutlook_2013.pdf

[2] Solar Energy Industry Association, "Solar Industry Data”, 2014, [Online] Available: http://www.seia.org/research-resources/solar-industry-data

[3] Palma-Behnke, R., Benavides, C., Lanas, F., Severino, B., Reyes, L.; Llanos, J., Saez, D., A Microgrid Energy Management System Based on the Rolling Horizon Strategy, IEEE Transactions on Smart Grid, Volume 4, Number 2, June 2013, Pages 996-1006

[4] Amjad Anvari-Moghaddam, Hassan Monsef, Ashkan Rahimi-Kian, Cost-effective and comfort-aware residential energy management under different pricing schemes and weather conditions, Energy and Buildings, Volume 86, January 2015, Pages 782-793 
[5] Palensky P., Dietrich D., Demand Side Management: Demand Response, Intelligent Energy Systems, and Smart Loads, IEEE Transactions on Industrial Informatics, Volume 7, Number 3, Aug. 2011, Pages 381-388

[6] Ye Yang, Hui Li, Aichhorn A.; Jianping Zheng, Greenleaf, M., Sizing Strategy of Distributed Battery Storage System With High Penetration of Photovoltaic for Voltage Regulation and Peak Load Shaving, IEEE Transactions on Smart Grid, Volume 5, Number 2, March 2014, Pages 982-991

[7] Sungjin Lee; Beom Kwon; Sanghoon Lee, Joint Energy Management System of Electric Supply and Demand in Houses and Buildings, IEEE Transactions on Power Systems, Volume 29, Number 6, Nov. 2014, Pages 2804-2812

[8] Susanna Mocci, Nicola Natale, Fabrizio Pilo, Simona Ruggeri, Demand side integration in LV smart grids with multi-agent control system, Electric Power Systems Research, Volume 125, August 2015, Pages 23-33

[9] Zhu Wang, Lingfeng Wang, Anastasios I. Dounis, Rui Yang, Integration of plug-in hybrid electric vehicles into energy and comfort management for smart building, Energy and Buildings, Volume 47, April 2012, Pages 260-266

[10] Yubo Wang; Sheikh O., Boyang Hu, Chi-Cheng Chu, Gadh R., Integration of V2H/V2G hybrid system for demand response in distribution network, 2014 IEEE International Conference on Smart Grid Communications, Nov. 2014, Pages 812-817

[11] Ota Y., Taniguchi, H., Nakajima, T., Liyanage K.M., Baba, J., Yokoyama A., Autonomous Distributed V2G (Vehicle-to-Grid) Satisfying Scheduled Charging, IEEE Transactions on Smart Grid, Volume 3, Number 1, March 2012, Pages 559-564

[12] Yubo Wang; Nazaripouya, H.; Chi-Cheng Chu; Gadh, R.; Pota, H.R., Vehicle-to-grid automatic load sharing with driver preference in micro-grids, 2014 IEEE PES Innovative Smart Grid Technologies Conference Europe, Oct. 2014, Pages1-6

[13] Alessandro Pensini, Claus N. Rasmussen, Willett Kempton, Economic analysis of using excess renewable electricity to displace heating fuels, Applied Energy, Volume 131, 15 October 2014, Pages 530-543

[14] Erdinc O., Paterakis N.G., Mendes T.D.P., Bakirtzis A.G., Catalao J.P.S., Smart Household Operation Considering Bi-Directional EV and ESS Utilization by Real-Time Pricing-Based DR, IEEE Transactions on Smart Grid, Volume 6, Number 3, May 2015, Pages 1281-1291 
[15] Petra Mesarić, Slavko Krajcar, Home Demand Side Management Integrated with Electric Vehicles and Renewable Energy Sources, Energy and Buildings, Available online 3 September 2015

[16] Aurélie Chabaud, Julien Eynard, Stéphane Grieu, A new approach to energy resources management in a grid-connected building equipped with energy production and storage systems: A case study in the south of France, Energy and Buildings, Volume 99, 15 July 2015, Pages 9-31

[17] Wenbo Shi, Na Li, Xiaorong Xie, Chi-Cheng Chu, Gadh R., Optimal Residential Demand Response in Distribution Networks, IEEE Journal on Selected Areas in Communications, Volume 32, Number 7, July 2014, Pages 1441-1450

[18] Malysz P., Sirouspour S., Emadi A., An Optimal Energy Storage Control Strategy for Grid-connected Microgrids, IEEE Transactions on Smart Grid, Volume 5, Number 4, July 2014, Pages 1785-1796

[19] Bai X., Qiao W., Robust Optimization for Bidirectional Dispatch Coordination of Large-Scale V2G, IEEE Transactions on Smart Grid, Volume 6, Number 4, July 2015, Pages 1944-1954

[20] Yu Zhang, Gatsis N., Giannakis G.B., Robust Energy Management for Microgrids With High-Penetration Renewables, IEEE Transactions on Sustainable Energy, Volume 4, Number 4, Oct. 2013, Pages $944-953$

[21] Qianfan Wang, Jianhui Wang, Yongpei Guan, Price-Based Unit Commitment With Wind Power Utilization Constraints, IEEE Transactions on Power Systems, Volume 28, Number 3, Aug. 2013, Pages 2718-2726

[22] Hieu Trung Nguyen, Long Bao Le, Optimal energy management for building microgrid with constrained renewable energy utilization, 2014 IEEE International Conference on Smart Grid Communications, Nov. 2014, Pages 133-138

[23] Garcia P., Garcia C.A., Fernandez L.M., Llorens F., Jurado, F., ANFIS-Based Control of a Grid-Connected Hybrid System Integrating Renewable Energies, Hydrogen and Batteries, IEEE Transactions on Industrial Informatics, Volume 10, Number 2, May 2014, Pages 1107-1117

[24] O. Erdinc, O. Elma, M. Uzunoglu, U.S. Selamogullari, B. Vural, E. Ugur, A.R. Boynuegri, S. Dusmez, Experimental performance assessment of an online energy management strategy for varying renewable power production suppression, International Journal of Hydrogen Energy, Volume 37, Issue 6, March 2012, Pages 4737-4748 
[25] Abdollah Kavousi-Fard, Alireza Abunasri, Alireza Zare, Rasool Hoseinzadeh, Impact of plug-in hybrid electric vehicles charging demand on the optimal energy management of renewable micro-grids, Energy, Volume 78, 15 December 2014, Pages 904-915

[26] Zhanbo Xu, Xiaohong Guan, Qing-Shan Jia, Jiang Wu, Dai Wang, Siyun Chen, Performance Analysis and Comparison on Energy Storage Devices for Smart Building Energy Management, IEEE Transactions on Smart Grid, Volume 3, Number 4, Dec. 2012, Pages 2136-2147

[27] Conell University Facilities Service, Real Time Building Utility Use Data, [Online]. Available: http://portal.emcs.cornell.edu/

[28] SMERC, Ackerman PV project, [online] Available: https://evsmartplug.net/smartgrid/ackerman_pv/

[29] Ching-Yen Chung, Shepelev, A., Qiu, C., Chi-Cheng Chu, Gadh, R., Design of RFID mesh network for Electric Vehicle smart charging infrastructure, 2013 IEEE International Conference on RFID-Technologies and Applications, Sept. 2013, Pages1-6

[30] B. Wang, B. Hu, C. Qiu, P. Chu, and R. Gadh, EV charging algorithm implementation with user price preference, 2015 IEEE Innovative Smart Grid Technologies Conference, Feb. 2015, Pages 1-5

[31] CHAdeMO society, Basic function of quick charger for the electric vehicle, Tech. Rep., 2012.

[32] Junhao Lin, Ka-Cheong Leung, Li, V.O.K., Optimal Scheduling With Vehicle-to-Grid Regulation Service, IEEE Internet of Things Journal, Volume 1, Number 6, Dec. 2014, Pages 556-569

[33] Sortomme, E., El-Sharkawi, M.A., Optimal Scheduling of Vehicle-to-Grid Energy and Ancillary Services, IEEE Transactions on Smart Grid, Volume 3, Number 1, March 2012, Pages 351-359

[34] Chengke Zhou, Kejun Qian, Allan M., Wenjun Zhou, Modeling of the Cost of EV Battery Wear Due to V2G Application in Power Systems, IEEE Transactions on Energy Conversion, Volume 26, Number 4, Dec. 2011, Pages 1041-1050,

[35] A. Shapiro, D. Dentcheva, and A. P. Ruszczynski, Lectures on Stochastic Programming: Modeling and Theory, 2nd Edition, SIAM, 2014

[36] S. Ahmed, A. Shapiro, The Sample Average Approximation Method for Stochastic Programs With Integer Recourse, ISyE, Georgia Institute of Technology, Atlanta, GA, Feb. 2002, Tech. Rep 
[37] Silverman, Bernard W, Density estimation for statistics and data analysis, 1986, CRC press, Boca Raton, Florida

[38] SAE International, SAE standard on EV charging connector. Oct. 2012

[39] Nissan Motor Company, "Vehicle to Home” Electricity Supply System, 2014, [Online] Available: http://www.nissan-global.com/EN/TECHNOLOGY/OVERVIEW/vehicle_to_home.html

[40] Gurobi Optimization Inc, 2015, [Online] Available: http://www.gurobi.com/

[41] California solar statistics, 2015, [Online] Available: http://www.californiasolarstatistics.ca.gov/

[42] Nykvist, Bjorn, and Mans Nilsson., Rapidly falling costs of battery packs for electric vehicles. Nature Climate Change, Volume 5, Issue 4, April 2015, Pages 329-332

[43] Song Chen, Peng Li, David Brady, Brad Lehman, Determining the optimum grid-connected photovoltaic inverter size, Solar Energy, Volume 87, January 2013, Pages 96-116

[44] V2G-Sim, 2015, [Online] Available: http://v2gsim.lbl.gov/ 